\title{
An Interval-Censored Model for Predicting Myopic Regression after Laser In Situ Keratomileusis
}

\author{
Yun-I Chen, ${ }^{1,2,3}$ Kuo-Liong Chien, ${ }^{2}$ I-Jong Wang, ${ }^{3}$ Amy Ming-Fang Yen, ${ }^{2}$ Li-Sheng Chen, ${ }^{2}$ \\ Pi-Jung Lin, ${ }^{4}$ and Tony Hsiu-Hsi Chen ${ }^{2,5,6}$
}

Purpose. A time-varying statistical model was proposed to predict the risk of regression toward myopia after laser in situ keratomileusis (LASIK) and to identify significant predictors within a time frame.

Methods. A total of 615 eyes of 311 patients derived from a retrospective cohort who underwent LASIK in 2003 were analyzed. Refraction outcomes were recorded at 1 day, 1 week, and $1,3,6,9$, and 12 months or longer after LASIK. A crossvalidated design was used, to split data into trained $(n=308)$ and validated $(n=307)$ data sets. These data sets were used in an interval-censored model to predict the probability of regression toward myopia and to assess the predictors including demographic features and preoperative and postoperative variables.

Results. Myopia regression was observed in 164 (26.7\%) of 615 eyes during the follow-up period of 12 months or longer after LASIK. Significant predictors for myopia regression after LASIK included preoperative manifest spherical equivalent $(P=<0.0001)$, mean preoperative central corneal curvature $(P=0.001)$, size of optic zone $(P=0.0043)$, undercorrection $(P=0.04)$, and age $(P=0.0734)$. The risk of regression toward myopia after LASIK increased rapidly within 1 month, slowed down between 1 and 6 months, and became steady after 6 months, regardless of risk group. The risk of myopia regression up to 6 months after LASIK was $21 \%$ in average-risk eyes (based on all eyes).

Conclusions. The proposed interval-censored model was useful not only for predicting the probability of myopia regression after LASIK but also for identifying the evolution of patients within low, moderate, and high-risk groups. (Invest Ophthalmol Vis Sci. 2007;48:3516-3523) DOI:10.1167/iovs.06-1044

$\mathrm{A}$ s both prevalence and severity of myopia have been noted over the past two decades in Taiwanese schoolchildren, ${ }^{1}$ treatment with LASIK (laser in situ keratomileusis) has increasingly gained attention. However, the incidence of regression toward myopia (hereafter, myopia regression) after LASIK var-

From the ${ }^{1}$ Department of Ophthalmology, Taipei Hospital, Taipei, Taiwan; the ${ }^{2}$ Institute of Preventive Medicine, the ${ }^{5}$ Centre of Biostatistics Consultation, and the ${ }^{6}$ Division of Biostatistics, Graduate Institute of Epidemiology, College of Public Health, and the ${ }^{3}$ Department of Ophthalmology, National Taiwan University, Taipei, Taiwan; and the ${ }^{4}$ Universal Eye Center, Taipei, Taiwan.

Submitted for publication September 4, 2006; revised March 18 and April 12, 2007; accepted June 11, 2007.

Disclosure: Y.-I. Chen, None; K.-L. Chien, None; I.-J. Wang, None; A.M.-F. Yen, None; L.-S. Chen, None; P.-J. Lin, None; T.H.-H. Chen, None

The publication costs of this article were defrayed in part by page charge payment. This article must therefore be marked "advertisement" in accordance with 18 U.S.C. $\$ 1734$ solely to indicate this fact.

Corresponding author: Tony Hsiu-Hsi Chen, Division of Biostatistics, Graduate Institute of Epidemiology, Centre of Biostatistics Consultation, and Institute of Preventive Medicine, College of Public Health, National Taiwan University, Room 521, No. 17, Hsuchow Road, Taipei 100, Taiwan; chenlin@ntu.edu.tw. ies across studies, ranging from $5.5 \%$ to $27.7 \% .^{2-7}$ Most of the previous studies in which investigators identified clinical correlates responsible for refractive outcome after LASIK were focused only on cases involving retreatment, ${ }^{2,4,8-10}$ rather than on all patients with different degrees of regression toward myopia, from mild to severe. However, patients' tolerance of residual myopia and their ophthalmologists' threshold for determining the need for retreatment play vital roles in their decisions for retreatments. ${ }^{4}$ As retreatment cases may represent only a small proportion of patients who had severe myopia regression after LASIK, this selection bias may lead to the failure of completely identifying clinical correlates related to myopia regression and may underestimate the risk of regression.

In addition to including all cases with myopia regression, it is also valuable to throw light on whether and when myopia regression may occur after LASIK. However, it is very difficult, from a practical aspect, to know the exact time of onset of myopia regression after LASIK between two follow-up visits. An interval-censored model, ${ }^{11}$ considering the degree of myopia regression occurring during a specific time interval after LASIK, was proposed to tackle this problem. The purposes of this study, conducted in all identifiable cases of myopia regression after LASIK, were therefore (1) to report the risk of myopia regression within a time frame; (2) to identify significant predictors; and (3) to develop a time-varying predictive model for estimating the probability of myopia regression by different time intervals of follow-up based on (1) and (2).

\section{MeTHODS}

\section{Study Subjects}

Subjects were enrolled from a retrospective cohort consisting of 372 patients with 735 eyes undergoing LASIK for myopia and myopic astigmatism by one experienced surgeon (PJL) with an excimer laser (EC-5000; Nidek Co., Ltd., Gamagori, Japan) at a franchise of ophthalmology clinic (Universal Eye Center, Taipei, Taiwan) between June and December 2003. The study was conducted according to the tenets of the Declaration of Helsinki of the World Medical Association regarding scientific research on human subjects.

The criteria for LASIK eligibility consisted of (1) patients over 18 years of age with myopia or myopic astigmatism and with stable refraction for at least 1 year; (2) no autoimmune diseases (e.g., lupus, rheumatoid arthritis) or uncontrolled diabetes; (3) no degenerative, neurotrophic corneal disease or scarring; (4) no current pregnancy or nursing; (5) no HIV (human immunodeficiency virus) or hepatitis C virus infection; (6) no previous ocular trauma or surgery within 6 months of the LASIK procedure.

Nasally oriented hinged flaps were created with a microkeratome (MK-2000; Nidek Co., Ltd.) with a flap diameter ranging from 8.5 to 9.0 $\mathrm{mm}$ and a thickness of 130 and $160 \mu \mathrm{m}$. The eyes were treated within an optic zone of 5.3 to $6.0 \mathrm{~mm}$ plus an additional transition zone, $1 \mathrm{~mm}$ wider than the optic zone. The standard laser ablation nomogram was used. A pulse repetition rate of $30 \mathrm{~Hz}$ and a laser energy pulse between 105 and $135 \mathrm{~mJ}$ were the laser parameters. The goal of our nomogram was emmetropia in all eyes. The targeted correction was calculated 
according to a customized clinical nomogram based on the Nidek photorefractive keratectomy (PRK) computer algorithm. The PRK nomogram was customized based on the prior surgeon's experience with LASIK and the Nidek EC-5000 excimer laser. The targeted cylinder correction (minus cylinder format) entered into the computer was equal to the baseline cylinder by manifest refraction. The target spherical correction (minus-cylinder format) was equal to (greater than -10 D) or was increased by $2 \%(-6$ to $-10 \mathrm{D})$ or $5 \%$ (lower than $-6 \mathrm{D})$ by the amount of manifest spherical component (minus cylinder format), but was further reduced by $1 \%$ of the cylinder component if the target cylinder was more than $-3 \mathrm{D}$.

After the ablation, the corneal flap was irrigated with a physiologic saline solution and then repositioned in the stromal bed with a cannula. A dripping-wet sponge was brushed gently across the cornea, lifting the excess fluid from the interface. The flap was allowed to adhere for 2 minutes. The slit beam of the laser was used to inspect possible wrinkles and debris. Plastic shields were placed after the surgery. The surgical site was inspected again with a standard slit-lamp biomicroscope to ensure proper flap position after blinking.

The postoperative regimen included a topical dexamethasone/neomycin solution (Decaneomycin; Union, Taiwan) applied four times a day for the first week followed by the administration of $0.1 \%$ fluorometholone (Fluorometholone; Oasis, Taiwan) four times a day with tapering dose until the end of the month. Preservative-free artificial tears in polyvinyl alcohol $14 \mathrm{mg} / \mathrm{mL}$ (Refresh; Allergan, Irvine, CA) were used during the postoperative course, with the date of withdrawal suggested by the ophthalmologist.

After exclusion of eyes with previous ocular surgeries $(n=4)$, those undergoing surgery with complications $(n=7)$, those lacking complete preoperative measurement records $(n=23)$, those having postoperative visits for $<1$ month $(n=78)$, and those having postoperative complications such as SOS (sands of the Sahara) syndrome $(n=$ 8), 615 eyes ( 311 subjects) met the inclusion criteria and were used in the analysis.

\section{Variables and Outcome Measurement}

Preoperative variables consisted of age, sex, manifest refraction measured by a phoropter with fogging, nomogram-adjusted refraction, and cycloplegic refraction. Refraction was recorded as sphere, cylinder, axis, and spherical equivalent (KR-7100; Topcon, Tokyo, Japan); central corneal thickness (CCT; Corneo-Gage Plus; Sonogage, Cleveland, $\mathrm{OH}$ ); preoperative central corneal curvature (Mean K, KR 7100; Topcon); intraocular pressure (IOP); and Schirmer test II result (basic tear reflex). Also recorded were the variables calculated by the standard laser ablation nomograms, including the diameters of the optic zone (Size_OZ) and transition zone (Size_TZ, $1 \mathrm{~mm}$ wider than the optic zone), the ablation depths of the optic and transition zones, and corneal flap diameter and depth. Residual bed thickness (RBT) was calculated by subtracting the flap thickness and optic zone thickness from the CCT.

Patients were examined at subsequent time points: 1 day, 1 week, and $1,3,6,9$, and 12 months or longer after the surgery. Manifest refraction results were recorded at each visit.

Myopia regression was defined as a residual myopia of $-1.0 \mathrm{D}$ or greater, detected at the last visit, and a 0.5 -D or greater shift toward myopia during follow-up visits. The eyes were otherwise defined as nonregressive. Undercorrection was defined as a residual refraction error of $-1 \mathrm{D}$ or greater detected 1 week after LASIK.

To evaluate the influence of preoperative manifest refraction on postoperative refraction change, all eyes we stratified into three groups, in accordance with a previous study ${ }^{6}$ : group 1 (low myopia), baseline refraction below $-6.0 \mathrm{D}$; group 2 (moderate myopia), baseline refraction from $-6.0 \mathrm{D}$ to less than $-10 \mathrm{D}$; and group 3 (high myopia), baseline refraction greater than or equal to $-10 \mathrm{D}$.

Most of the eyes were treated with an optic zone of $6.0 \mathrm{~mm}$ or above ( 352 eyes), in light of two previous studies, ${ }^{12,13}$ and therefore the eyes were stratified into another two groups for assessment of the refraction change after LASIK: group 1, eyes with optic zone greater than or equal to $6.0 \mathrm{~mm}$; group 2, eyes with optic zone $<6.0 \mathrm{~mm}$.

To build up a predictive model for myopia regression, a crossvalidation design was used with random allocation of one eye to the trained data set and the other eye to the validated data set for those individuals with both eyes used in the study (304 subjects; 608 eyes). Seven other individuals with only one eye used were randomly allocated to the trained data set or the validated data set. Thus, there were 308 eyes used in the trained model and 307 eyes in the validated model.

The trained data set was first used to estimate regression coefficients (Table 2), which, together with the values of predictors from the validated data, were further used to predict occurrence of myopia regression, which was compared with the observed regression, to assess the difference between the observed and the predicted, yielding sensitivity and $1-$ specificity on which analysis of the receiver operating (ROC) curve was based. In principle, the smaller the difference the better internal validity the model was considered to show, by ROC curve (see below).

\section{Preprocessing and Selecting Highly Correlated Predictors for Myopia Regression}

To avoid high correlation between similar clinical correlates-the so-called multicollinearity problem-we checked the predictors with high correlation with each other and preselected one among them before performing the following multivariate analysis. We first dealt with the relationships of the variables with a linear function (e.g., MSE is a linear function of Msph and Mcyl (MSE $=$ Msph $+1 / 2$ Mcyl) (abbreviations defined in the next paragraph) by retaining only one of the variables. In the case of this example, we retained only MSE and dropped Msph and Mcyl. Similarly, diameter of the optic zone (Size_OZ) was retained and the diameter of the transition zone (Size_TZ) was dropped.

Pearson correlation analysis was further applied, to assess the extent of the correlations between any two independent variables. Three groups were identified in the light of ranking correlation: correlation coefficients greater than 0.6 (group 1, including manifest axis [Maxis], nomogram-adjusted axis [Nomo_axis] and cycloplegic adjusted axis [Caxis]); correlation coefficients greater than 0.8 (group 2, including MSE, Msph, nomogram-adjusted spherical equivalent [Nomo$\mathrm{SE}$ ], nomogram-adjusted sphere [Nomo_sph], cycloplegic spherical equivalent [CSE], cycloplegic spherical equivalent [Csph], depth of optic zone [Depth_OZ] and depth of transition zone [Depth_TZ]), and correlation coefficients equal to 0.9 (group 3 , including CCT and RBT).

According to the principle of reducing superfluous variables due to multicollinearity (high correlation between two variables) and taking into account clinical significance, we selected one variable from each study group: MSE for group 1, Maxis for group 2, and RBT for group 3. These three selected variables, together with the remaining variablesage, sex, preoperative central corneal curvature (Mean K), diameter of optic zone (Size_OZ), flap thickness and diameter, Schirmer test II result, intraocular pressure (IOP), residual bed thickness (RBT), and undercorrection-formed a constellation of predictors for myopia regression included in the following multivariable model.

\section{Statistical Analysis}

Student's $t$-test and ANOVA were used to assess the differences of relevant clinical correlates across two or three groups. Differences were considered statistically significant at $P<0.05$. To detect multicollinearity, we applied the Pearson product moment correlation analysis to examine the correlation between independent variables and to classify three groups in the light of correlation coefficients.

To guarantee that there was no multicollinearity problem among the variables in the final model, a multiple linear regression between independent variables and the final manifest refraction was performed to check whether the variance inflation factor (VIF), one of the indicators for detecting multicollinearity, was less than 10 . 
Because myopia regression was detected during follow-up visits without knowledge of the exact occurrence time, we adopted an interval-censored model underpinning the concept of the generalized model with complementary log-log transformation of the probability of myopia regression. A detailed description of the model has been provided by Collett. ${ }^{11}$

The model can be expressed as

$$
\begin{gathered}
\log \left[-\log \left(1-\pi_{i j}\right)\right]=\gamma_{j}+\operatorname{SCORE}_{i} \\
\pi_{i j}=1-\exp \left[-\exp \left(\gamma_{j}+\operatorname{SCORE}_{i}\right)\right],
\end{gathered}
$$

where $\pi_{i j}$ is the probability that the $i$ th subject will have myopia regression in the $j$ th period and $\gamma_{j}$ is the regression coefficient of the $j$ th follow-up period denoted by $\gamma_{1}, \gamma_{2}, \ldots, \gamma_{5}$, corresponding to five intervals between follow-up visits: 1 week to 1 month, 1 month to 3 months, 3 months to 6 months, 6 months to 9 months, 9 months to 12 months or longer after surgery. Note that since the interval between examinations was not equivalent, the variable "period" was treated as a categorical variable. The risks for the development of myopia regression in each period $\left(\gamma_{j}\right)$ were also estimated.

Score $_{i}$ represents the magnitude of the risk of having myopia regression based on clinical correlates, for the $i$ th subject. It can be written as follows

$$
\text { Score }=\alpha+\Sigma \beta X
$$

where $\alpha$ is the intercept of the predictive model, $X$ is a set of predictors, and $\beta$ is the corresponding regression coefficients that can be trained by the application of the interval-censored model using equations 1 and 2 with the trained data set.
To assess the adequacy of the predictive model, the receiver operating characteristic (ROC) curve was further used by plotting the sensitivity ( $y$-axis) against $1-$ specificity ( $x$-axis), both of which were calculated by cross-tabulating the predicted with the observed, using the trained and validated data sets, respectively, given a series of cutoff points based on scores in equation 2. The larger the area under the ROC, the better the internal validity of the model. Whether the lower limit of the $95 \%$ confidence interval (CI) for the area under the ROC curve was larger than $50 \%$ was used to assess statistical significance for the adequacy of the predictive model. Note that the calculations were performed for both the trained and validated data sets, but the result from the validated model is more important than that from the trained model, because the former was not involved in training regression coefficients. All statistical analyses were performed using the SAS 9.1 program for Windows.

\section{Results}

\section{Baseline Characteristics}

Table 1 presents the descriptive results of preoperative characteristics of patients. In the 311 patients, there was a preponderance of women $(n=242 ; 77.8 \%)$, and the mean age was $31.1 \pm 6.2$ (SD) years (interquartile range: 27,35 years). The mean manifest spherical equivalent refraction was $-7.08 \pm$ 2.49 (SD) D (interquartile range: $-8.88,-5.13 \mathrm{D}$ ). The mean ablation depth of the optic zone was $99.27 \pm 26.95 \mu \mathrm{m}$ (interquartile range: $82.2,120.6 \mu \mathrm{m}$ ) with a mean optic zone diameter of $5.81 \pm 0.34 \mathrm{~mm}$ (interquartile range: $5.6,6.0 \mathrm{~mm}$ ).

\section{Refraction Change after LASIK}

Myopia regression after LASIK was observed in 164 (26.7\%) of

\begin{tabular}{|c|c|c|c|c|}
\hline Variable & Mean & SD & Lower Quartile & Upper Quartile \\
\hline Age $(y)$ & 31.1 & 6.2 & 27 & 35 \\
\hline \multicolumn{5}{|l|}{ Manifest refraction } \\
\hline Msph (D) & -6.61 & 2.40 & -8.50 & -4.75 \\
\hline Mcyl (D) & -0.95 & 0.76 & -1.25 & -0.50 \\
\hline Maxis (deg) & 104.88 & 79.99 & 15.00 & 170.00 \\
\hline $\operatorname{MSE}(\mathrm{D})$ & -7.08 & 2.49 & -8.88 & -5.13 \\
\hline \multicolumn{5}{|l|}{ Cycloplegic refraction } \\
\hline Csph (D) & -6.39 & 2.84 & -8.00 & -4.50 \\
\hline Ccyl (D) & -1.13 & 3.11 & -1.25 & -0.50 \\
\hline Caxis (deg) & 96.95 & 74.02 & 11.00 & 170.00 \\
\hline $\operatorname{CSE}(\mathrm{D})$ & -6.95 & 3.24 & -8.75 & -4.88 \\
\hline \multicolumn{5}{|l|}{ Nomogram-adjusted refraction } \\
\hline Nomo_sph (D) & -6.73 & 2.39 & -8.50 & -5.00 \\
\hline Nomo_cyl (D) & -0.93 & 0.83 & -1.25 & -0.50 \\
\hline Nomo_axis (deg) & 102.61 & 182.04 & 5.00 & 170.00 \\
\hline NomoSE (D) & -7.19 & 2.50 & -9.00 & -5.25 \\
\hline MeanK (D) & 43.67 & 1.95 & 42.83 & 44.63 \\
\hline Flap_thick $(\mu \mathrm{m})$ & 131.71 & 6.96 & 130.00 & 130.00 \\
\hline Flap_diameter (mm) & 8.97 & 0.17 & 9.00 & 9.00 \\
\hline Size_OZ $(\mathrm{mm})$ & 5.81 & 0.34 & 5.60 & 6.00 \\
\hline Size_TZ $(\mathrm{mm})$ & 6.83 & 0.37 & 6.70 & 7.00 \\
\hline Depth_OZ $(\mu \mathrm{m})$ & 99.27 & 26.95 & 82.20 & 120.60 \\
\hline Depth_TZ $(\mu \mathrm{m})$ & 17.15 & 6.69 & 13.50 & 20.40 \\
\hline Central corneal thickness (CCT) $(\mu \mathrm{m})$ & 546.75 & 55.81 & 529.00 & 571.00 \\
\hline $\mathrm{RBT}(\mu \mathrm{m})$ & 315.76 & 60.93 & 287.00 & 344.20 \\
\hline IOP (mm Hg) & 15.53 & 6 & 13.00 & 17.00 \\
\hline Schirmer test (mm) & 20.35 & 10.31 & 14.00 & 26.00 \\
\hline
\end{tabular}
615 eyes during a 12-month or longer follow-up period. Among

TABle 1. Basic Characteristics of Preoperative Variables

Msph, manifest sphere; Mcyl, manifest cylinder; Maxis, manifest axis; MSE, manifest spherical equivalent; Csph, cycloplegic sphere; Ccyl, cycloplegic cylinder; Caxis, cycloplegic axis; CSE, cycloplegic spherical equivalent; Nomo_sph, nomogram-adjusted sphere; Nomo_cyl, nomogramadjusted cylinder; Nomo_axis, nomogram-adjusted axis; NomoSE, nomogram-adjusted spherical equivalent; Mean K, mean keratometry; Flap_thick, flap thickness; Size_OZ, size of optic zone; Size_TZ, size of transition zone; Depth_OZ, depth of optic zone; Depth_TZ, depth of transition zone; CCT, central corneal thickness; RBT, residual bed thickness. 
Figure 1. The comparisons of mean spherical equivalent across three groups stratified by the degree of myopia over, with significance $\left({ }^{*} P<\right.$ 0.05 ) assessed by analysis of variance (ANOVA).

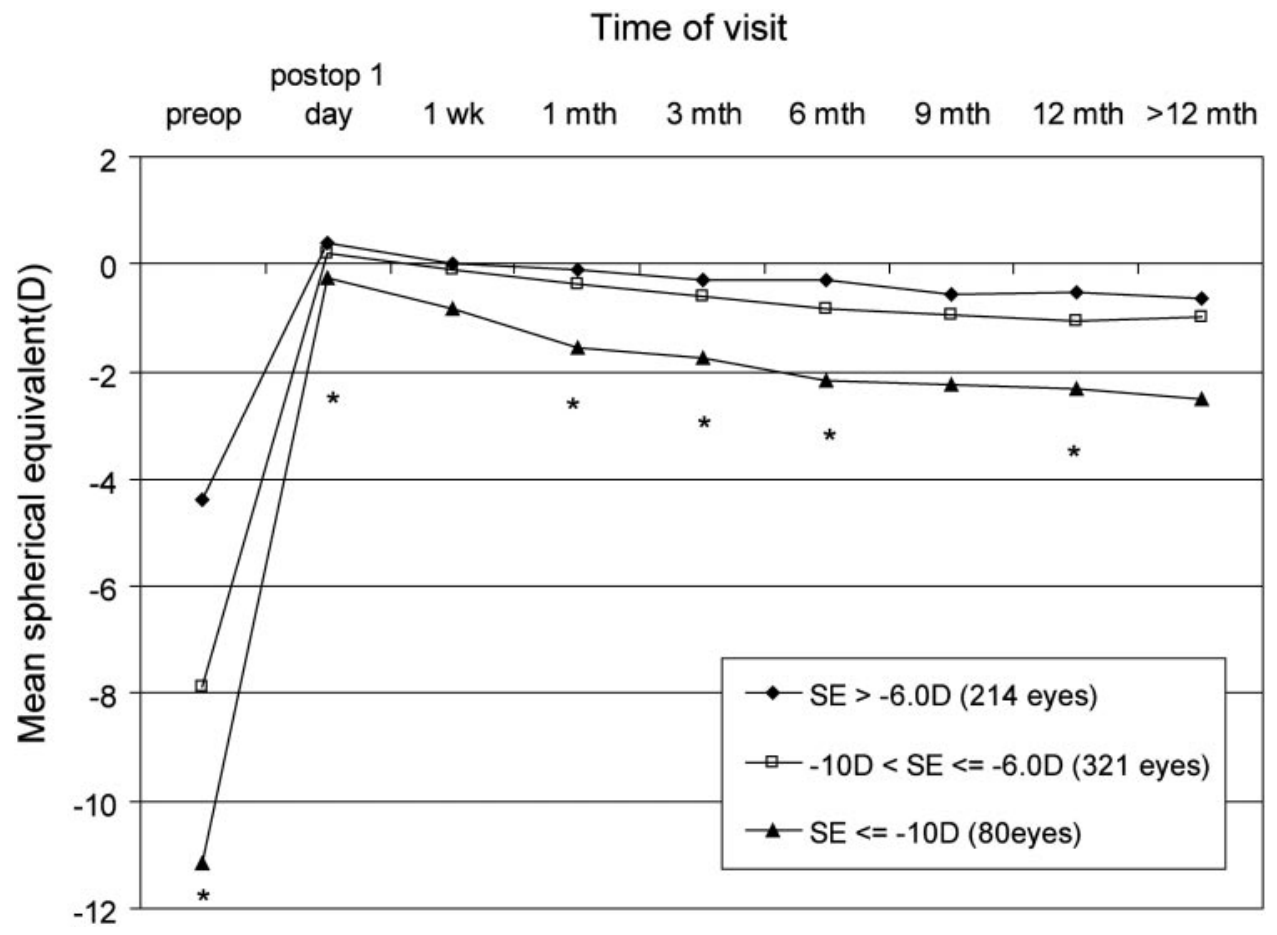

the 81 eyes with undercorrection, 47 (58\%) eyes were susceptible to regression. The preoperative mean manifest spherical equivalent refraction in undercorrected eyes was $-8.88 \pm 2.56$ $\mathrm{D}$ and was $-6.81 \pm 2.37 \mathrm{D}$ in adequately corrected eyes.

Greater preoperative myopia led to a higher likelihood of having myopia regression after LASIK. Statistically significant differences were found 1 day and 1, 3, 6, and 12 months after surgery across three groups (low myopia with baseline refraction below $-6.0 \mathrm{D}$, moderate myopia with baseline refraction from -6.0 to $-10.0 \mathrm{D}$, high myopia with baseline refraction greater than $-10 \mathrm{D}$; Fig. 1).

Eyes with smaller optic zones had a higher likelihood of having a greater amount of regression toward myopia after
LASIK. Statistically significant differences between eyes with a small optic zone (diameter $<6.0 \mathrm{~mm}$ ) and those with a large optic zone (diameter $\geq 6.0 \mathrm{~mm}$ ) were found $1,6,9$, and 12 months after surgery (Fig. 2).

\section{Time-Varying Predicted Probability for Myopia Regression with Predictors}

Table 2 shows the results of multivariate analysis in an intervalcensored model. Significant factors identified in the multivariate analysis included manifest spherical equivalent $(\beta=-0.3570$, $P=<0.0001)$, mean preoperative corneal curvature $(\beta=$ $-0.1807, P=0.0009)$, diameter of optic zone $(\beta=-0.6593$,
FIGURE 2. The comparisons of mean spherical equivalent between the two groups stratified by diameter of optic zone, with significant result $\left({ }^{*} P<\right.$ 0.05 ) assessed by independent $t$-test.

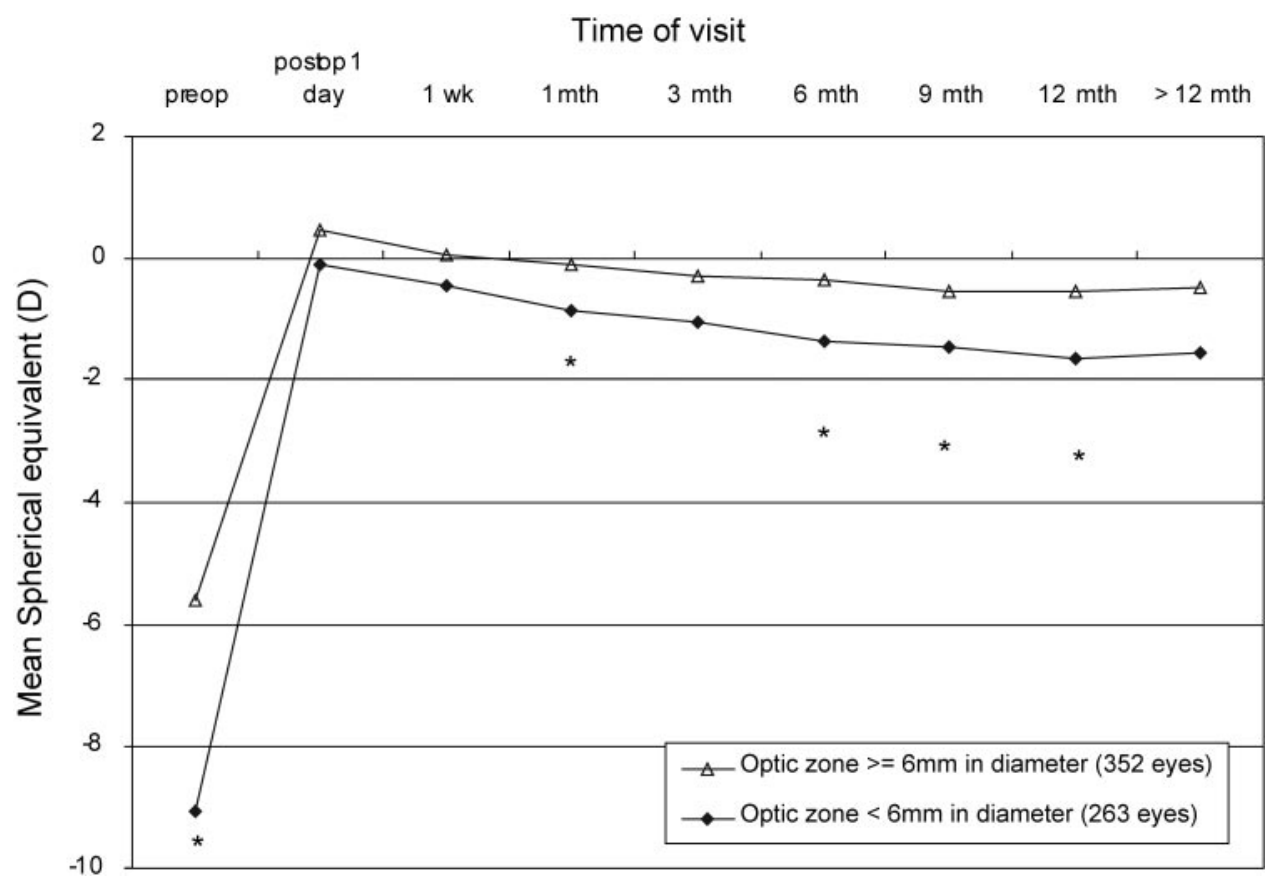


TABLE 2. Estimated Results of Multivariate Analysis in an IntervalCensored Model

\begin{tabular}{lccc}
\hline \multicolumn{1}{c}{ Variable } & $\begin{array}{c}\text { Regression } \\
\text { Coefficient }\end{array}$ & SE & $\boldsymbol{P}$ (Wald Test) \\
\hline Intercept & 5.9856 & 2.8238 & 0.0340 \\
Period $(\gamma)$ & & & 0.0219 \\
$\quad$ Period 1 & 0 & & \\
Period 2 & -0.9541 & 0.3496 & \\
Period 3 & -0.2530 & 0.3280 & \\
Period 4 & -0.8615 & 0.5998 & \\
Period 5 & -0.8945 & 0.6016 & 0.0009 \\
MSE $\left(\beta_{1}\right)$ & -0.3570 & 0.0505 & 0.0043 \\
Mean K $\left(\beta_{2}\right)$ & -0.1807 & 0.0544 & 0.0396 \\
Size_OZ $\left(\beta_{3}\right)$ & -0.6593 & 0.2311 & 0.0734 \\
Undercorrection $\left(\beta_{4}\right)$ & 0.5530 & 0.2688 & \\
Age $\left(\beta_{5}\right)$ & 0.0297 & 0.0166 & \\
\hline
\end{tabular}

Periods 1 to 5 represent 1 week to 1 month, 1 month to 3 months, 3 months to 6 months, 6 months to 9 months, and 9 months to more than 12 months after surgery, respectively.

$P=0.0043)$, undercorrection $(\beta=0.5530, P=0.0396)$, and age $(\beta=0.0297, P=0.0734)$. The risks of myopia regression in the five periods $\left(\gamma_{1}, \gamma_{2}, \ldots, \gamma_{5}\right)$ are also shown in Table 2.

Using our interval-censored model, Table 3 shows the predicted probability of myopia regression in each period and cumulative probability up to a certain period using three selected eyes, representing groups with low, moderate, or high myopia. Taking the first case as an example, the score is calculated as:

intercept $+\beta_{1} \times$ MSE $+\beta_{2} \times$ Mean $\mathrm{K}+\beta_{3} \times$ size_OZ $+\beta_{4}$

$$
\begin{aligned}
& \times \text { undercorrection }+\beta_{5} \times \text { age }=5.9856+(-0.3570) \\
& \cdot(-13.625)+(-0.1807) \cdot 44.24+(-0.6593) \\
& \cdot 5.5+0.5530 \cdot 0+0.0297 \cdot 23=-0.0875,
\end{aligned}
$$

where $\beta_{1}, \beta_{2}, \ldots, \beta_{5}$ are the coefficients for each period in Table 2. Calculation of equation 1 shows that the predicted probability $\left(\pi_{1}\right)$ of myopia regression in the first period (from 1 week to 1 month after LASIK) is

$$
\pi_{1}=1-\exp \left\{-\left[\exp \left(\gamma_{1}+\mathrm{SCORE}\right)\right]\right\}=0.6002,
$$

where $\gamma_{1}$ is the regression coefficient of the first follow-up period (Table 3 ). The predicted probability of other periods $\left(\pi_{2}, \pi_{3}, \pi_{4}\right.$ and $\left.{ }_{\pi 5}\right)$ for the same patient can be calculated in a similar manner. Therefore, the cumulative probability of myopia regression until period 5 is equivalent to

$\pi($ recurrence in period 1$)$

$+\pi($ recurrence in period 2 and no recurrence in period 1$)$

$$
+\ldots+\pi(\text { recurrence in period } 5
$$

no recurrence in period $1, \ldots$, and period 4)

$$
\begin{gathered}
=\pi_{1}+\pi_{2}\left(1-\pi_{1}\right)+\pi_{3}\left(1-\pi_{1}\right)\left(1-\pi_{2}\right) \\
+\pi_{4}\left(1-\pi_{1}\right)\left(1-\pi_{2}\right)\left(1-\pi_{3}\right)+\pi_{5}\left(1-\pi_{1}\right)\left(1-\pi_{2}\right) \\
\times\left(1-\pi_{3}\right)\left(1-\pi_{4}\right)=1-\left[\left(1-\pi_{1}\right)\left(1-\pi_{2}\right)\right. \\
\left.\times\left(1-\pi_{3}\right)\left(1-\pi_{4}\right)\left(1-\pi_{5}\right)\right]=1-[(1-0.6002)(1-0.2975) \\
\times(1-0.5092)(1-0.3211)(1-0.3126)]=0.9357 .
\end{gathered}
$$

\begin{tabular}{|c|c|c|c|c|c|c|c|c|c|}
\hline Eye & MSE & MEANK & Size_OZ & $\begin{array}{c}\text { Under } \\
\text { correction* }\end{array}$ & Age & Score & Period $†$ & Probability $\ddagger$ & $\begin{array}{l}\text { Cumulative } \\
\text { Probability } \$\end{array}$ \\
\hline \multirow[t]{5}{*}{1} & -13.625 & 44.24 & 5.5 & 0 & 23 & -0.0875 & 1 & 0.6002 & 0.6002 \\
\hline & & & & & & & 2 & 0.2975 & 0.7191 \\
\hline & & & & & & & 3 & 0.5092 & 0.8621 \\
\hline & & & & & & & 4 & 0.3211 & 0.9064 \\
\hline & & & & & & & 5 & 0.3126 & 0.9357 \\
\hline \multirow[t]{5}{*}{2} & -8.25 & 42.75 & 5.5 & 0 & 35 & -1.3807 & 1 & 0.2223 & 0.2223 \\
\hline & & & & & & & 2 & 0.0923 & 0.2941 \\
\hline & & & & & & & 3 & 0.1773 & 0.4193 \\
\hline & & & & & & & 4 & 0.1008 & 0.4778 \\
\hline & & & & & & & 5 & 0.0977 & 0.5288 \\
\hline \multirow[t]{5}{*}{3} & -2.75 & 44.69 & 6.5 & 0 & 23 & -4.7105 & 1 & 0.0091 & 0.0091 \\
\hline & & & & & & & 2 & 0.0035 & 0.0124 \\
\hline & & & & & & & 3 & 0.0071 & 0.0194 \\
\hline & & & & & & & 4 & 0.0039 & 0.0232 \\
\hline & & & & & & & 5 & 0.0017 & 0.0248 \\
\hline \multirow[t]{5}{*}{ All eyes (mean) } & -7.08 & 43.67 & 5.81 & 0.13171 & 31 & -2.2094 & 1 & 0.1040 & 0.1040 \\
\hline & & & & & & & 2 & 0.0414 & 0.1411 \\
\hline & & & & & & & 3 & 0.0817 & 0.2113 \\
\hline & & & & & & & 4 & 0.0453 & 0.2470 \\
\hline & & & & & & & 5 & 0.0439 & 0.2801 \\
\hline
\end{tabular}

(Table 3). The eyes with a higher score had a greater probability of myopia regression. The predicted probabilities of average risk based on the mean of all eyes are also shown in Table 3 . Figure 3 shows the cumulative probability up to a a certain period in the three groups. The risk of myopia regression

TABLE 3. Variables and Time-Varying Predicted Probabilities in Three Selected Cases in an Interval-Censored Model

* Undercorrection of 1 was defined as a residual refraction error of -1 D or greater, detected 1 week after LASIK. Otherwise, undercorrection was 0 .

+ Periods 1 to 5 are as described in Table 2

$\neq$ Conditional probability of myopia regression in each period.

$\int$ Cumulative probability is the probability of myopia regression up to a certain period. 


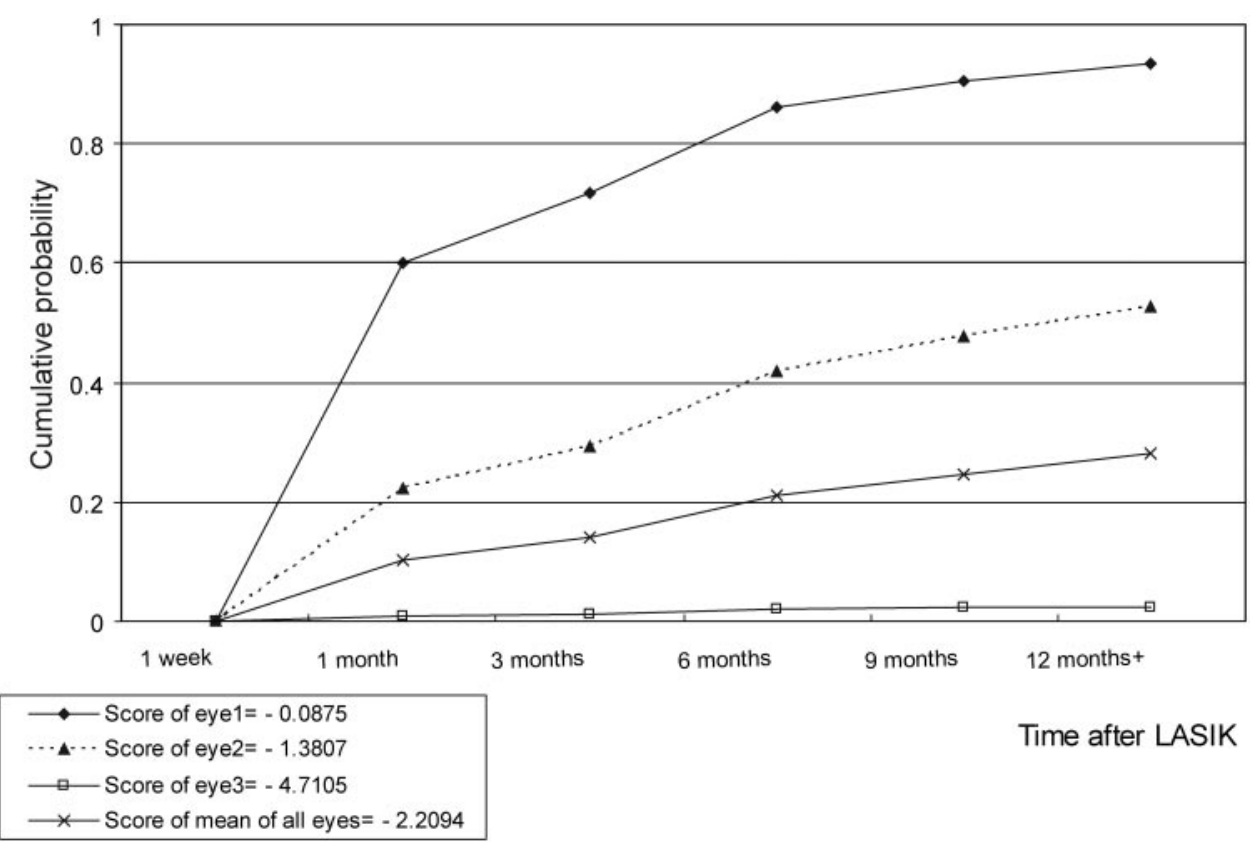

Figure 3. Cumulative risk of myopia regression for the overall mean of all eyes and three selected cases in an interval-censored model.

increased rapidly within a month, slowed between 1 and 6 months, and became steady after 6 months, regardless of risk group. The risks of myopia regression up to 6 months after LASIK were $86 \%, 42 \%$, and $2 \%$ for high, moderate, and low risks, respectively. The corresponding risk estimate for the average risk based on all eyes was $21 \%$.

\section{Model Validation of Predictive Model}

The validity of this model was tested by cross-validation using the ROC curve. The area under the curve of the intervalcensored model was $82.96 \%$ (95\% CI: $77.77 \%-88.16 \%)$ in the trained group and $78.43 \%$ (95\% CI: $73.04 \%-83.82 \%)$ in the validated group (Fig. 4). The lower limit of the $95 \%$ CI of the area under the ROC were significantly higher than $50 \%$ in the validated group, which suggests that the predicted validity of our model was good.

\section{Discussion}

\section{Variation of Regression and Retreatment Rate}

The complete ascertainment of myopia regression after LASIK, together with the application of an interval-censored model suggests that the risk of myopia regression after LASIK is highly dependent on the definition and inclusion criteria of myopia regression and the reported follow-up time after LASIK. This dependency on inclusion criteria accounts for why myopia regression after LASIK reported in previous studies has ranged from $5.5 \%$ to $27.7 \% .^{2-6}$ Lyle et al. ${ }^{2}$ reported 114 eyes re-treated for regression with an overall regression rate of $5.5 \%$ in a large case study (114/2065 eyes with preoperative myopia of $-6.11 \pm 2.35 \mathrm{D})$. The lower rate may be attributable to the exclusion of the cases of regression that did not undergo retreatment. The definition of regression in Lyle et al. ${ }^{2}$ was a residual myopia of greater than -0.5 and a 0.25 -D or greater shift toward myopia between follow-ups except in the cases of undercorrection. Chayet et al. ${ }^{3}$ found a regression rate of $28 \%$ (13/47 eyes were retreated for regression) in patients with mean preoperative myopia of $-14.02 \pm 5.3 \mathrm{D}$ based on the definition of regression as a 0.25 -D or greater myopic shift occurred between follow-up visits. If we take different operative definitions of myopia regression in previous stud- ies $^{2,3,5,6,14,15}$ as a reference, our regression rate defined by a residual myopia of $-1.0 \mathrm{D}$ or greater and myopic shift by 0.5 $\mathrm{D}$ or greater during follow-up visits is $26.7 \%$, close to the $28.01 \%$ predicted value reported in Table 3 . The incidence of myopia regression found in our study is probably more comprehensive than that reported in the previous studies, because we analyzed the evolution of all cases undergoing LASIK by one surgeon instead of retreatment cases only.

The use of an interval-censored model provides a comparison of the predicted time of onset of myopia regression against the reported time frame available in the literature. The myopia regression rate after LASIK has been reported within 6 months after surgery in several studies, ${ }^{3,8,16-18}$ but only one study included follow-up to 1 to 2 years after surgery. ${ }^{2}$ In cases of high preoperative myopia, regression has been observed to peak within 6 months after the initial LASIK surgery and then to stabilize afterward. ${ }^{3,16}$ This finding was consistent with our estimated results based on the average-risk group. Cumulative risk for myopia regression increased from $10 \%$ within a month to $21 \%$ within 6 months after LASIK but then became stable (Table 3). In eyes with preoperative myopia of $-14 \mathrm{D}$, Chayet et al. ${ }^{3}$ reported a regression rate of $27.7 \%$ within 3 months after surgery that then stabilized between 3 and 6 months. Their result was higher than the $14 \%$ reported in our study (Table 3), because patients treated with LASIK in their study had a higher grade of myopia than did those in our study $(-14$ D vs. $-7.08 \mathrm{D})$. Perez-Santonja et al. ${ }^{16}$ reported a regression of $0.53 \mathrm{D}$ between 1 and 3 months after surgery in eyes with preoperative mean myopia of $-13 \mathrm{D}$, but no significant regression after 3 months after surgery. Based on Figure 2 in PerezSantonja et al., ${ }^{16}$ the regression rate when $-1 \mathrm{D}$ was used as a criterion 6 months after surgery was $\sim 19.56 \%$, which was close to the $21 \%$ in our study (Table 3 ).

\section{Predictors of Myopia Regression}

Factors reported to be associated with refractive outcome after LASIK vary from study to study, including preoperative refractive error, ${ }^{2-4,6,7,16,19-22}$ corneal curvature, ${ }^{2,3,14,16}$ corneal thickness, ${ }^{3,6,15,20}$ flap thickness, ${ }^{23-25}$ ablation depth, ${ }^{2}$ optic zone size, ${ }^{6,12}$ chronic dry eye, ${ }^{5}$ age, ${ }^{4}$ surgeon, ${ }^{4}$ IOP, ${ }^{2,16}$ and humidity. ${ }^{2}$ Using an interval-censored model, 


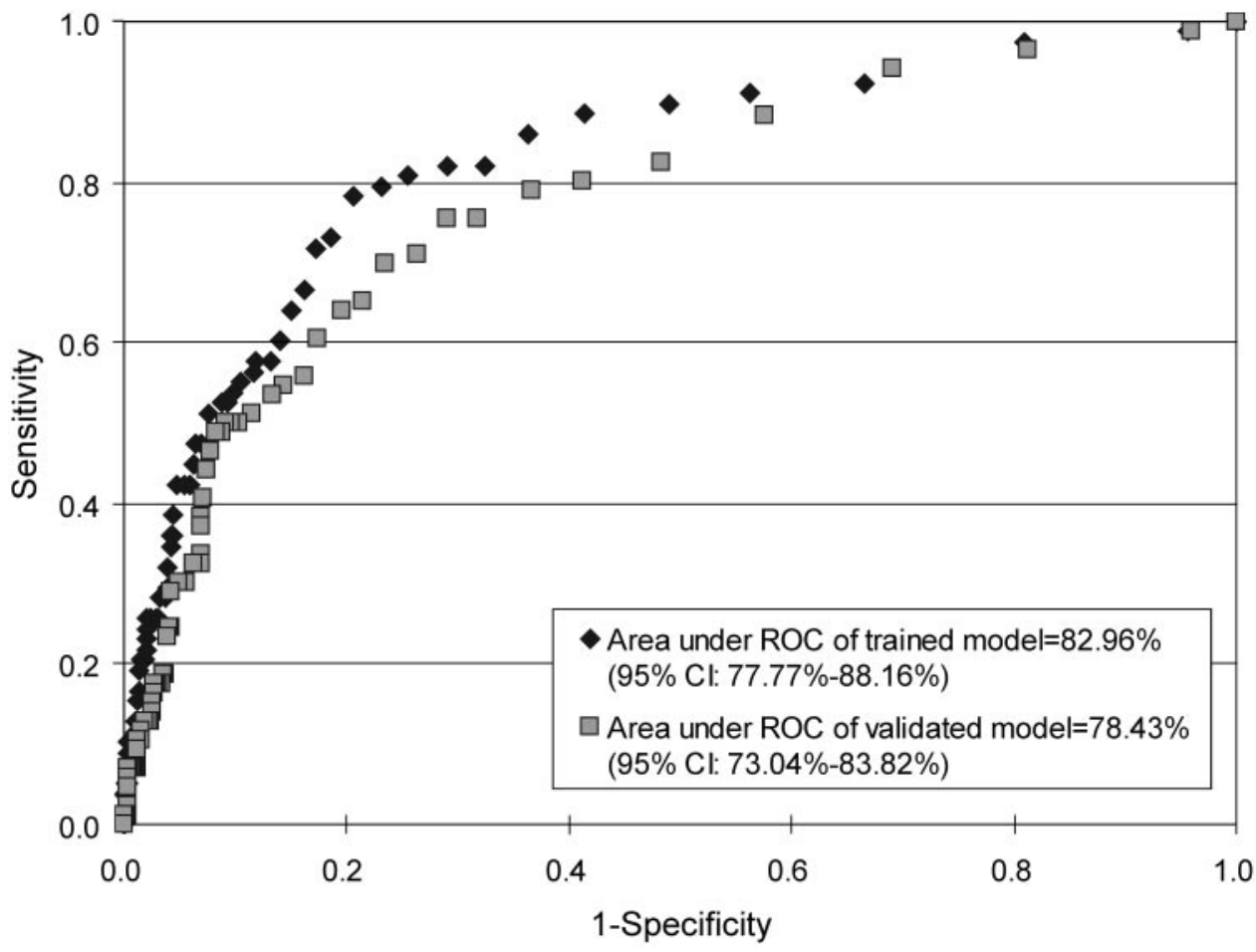

Figure 4. Area under ROC curves and $95 \% \mathrm{CI}$, based on the trained and the validation data sets, in an interval-censored model. we not only identified clinical correlates related to myopia regression but also predicted time-varying risk for myopia regression. We considered multicollinearity between variables and chose the appropriate ones, given the orthogonal properties between two of each. Significant predictors identified in multivariate analysis of the interval-censored model included manifest refraction, preoperative keratometry reading, size of optic zone, and undercorrection. Our significant predictors were consistent with those identified in the previous findings. Greater preoperative refraction ${ }^{2-4,6,7,16,19-22}$ increased the probability of myopia regression or retreatment; size of optic zone ${ }^{6,12,16}$ was associated with refractive outcome after LASIK; flatter preoperative keratometry was associated with a more myopic outcome after LASIK ${ }^{16}$; and preoperative keratometry $<43.5 \mathrm{D}$ predicted greater undercorrection (greater residual myopia shift at 3 months after surgery, according to the definition in Rao et al., which may contain some regressed eyes, according to our definition). ${ }^{14}$

Different from previous studies on retreatment after LASIK, which did not regard initial undercorrection cases as an independent variable, ${ }^{2,3,26}$ our study treated undercorrection as one of our independent variables in multivariate analysis instead and found that undercorrection led to a higher probability of myopia regression. However, a high association between undercorrection and preoperative MSE is probable. This probability raises a concern as to whether both, if included in the same model, may cause colinearity. However, as tested by the VIF, a method for detecting colinearity, no apparent colinearity problem was revealed and thus undercorrection and preoperative MSE should be thought of as two independent predictors of myopia regression.

\section{Usefulness of the Predictive Model}

In most previous studies, the assessment of the overall effects of multiple factors on outcome after LASIK have been addressed with only one or two factors, and very few studies have developed a model based on a series of predictors. Hu et al. ${ }^{4}$ developed a mathematical predictive formula for retreat- ment, with multiple variables including age, preoperative cycloplegic sphere, and surgeon. Our study had two characteristics that were different from those in Hu et al. ${ }^{4}$ : In their study, the risk of myopia regression was not reported in a longitudinal time frame, and only retreatment cases were included. A logistic regression model that treats retreatment or not as a binary variable (yes/no) predicted an $18 \%$ rate of retreatment, which is slightly lower than the predicted $28 \%$ rate a year after LASIK - the time when myopia regression reached a plateau. The higher rate in our study compared with that in $\mathrm{Hu}$ et al. was partly due to the inclusion of myopia regression in our study rather than only retreatment as in $\mathrm{Hu}$ et al. and also was partly due to different types of predictive models in our study (interval-censored model) and their study (logistic regression model). Another disparity is that the "surgeon factor" was taken into account in their study in which patients were treated by two surgeons who had variations in threshold for retreatment, whereas only one ophthalmologist was involved in our study. Our model focused on each eye rather than a subject (person) with cross-validated design by randomly selecting one eye from each subject to build up the predictive model. This method may eliminate the problem of correlation between both eyes of a patient.

An interval-censored model for predicting the risk of myopia regression with follow-up time after LASIK has never been developed before, to the best of our knowledge. The novel idea of applying an interval-censored model is to predict when and what sort of subjects may be at risk of myopia regression, corresponding to the evolution of refraction after LASIK over time. When the exact time point of the onset of myopia regression is not discernible, analysis with this model to examine occurrence between two visits, given a time frame, will be more accurate in identifying related risk factors and in predicting the probability of myopia regression after LASIK, which will be helpful in counseling patients during follow-up.

There are two limitations to our study. Since our outcomes were based on changes in refractive error, as measured by the optometer, the result in determining regression could also be 
influenced by the accommodation of the subject. Based on the biomechanical factors observed to accompany myopia regression, ${ }^{3,15,16}$ the definition of myopia regression would have been more valid if the measurement of the postoperative Mean $\mathrm{K}$ and CCT had been collected together. Second, one of our variables in the model is undercorrection, which is identified after surgery, and thus may limit the model's application in patient counseling before LASIK but is still useful for predicting the evolution of refraction during follow-up after LASIK.

In conclusion, we propose a useful time-varying predictive model to predict myopia regression after LASIK for myopia and myopic astigmatism. Information obtained from the predictive model enables a clinician to identify who has a high risk of myopia regression and to estimate the probability within the follow-up time frame more precisely.

\section{References}

1. Lin LL, Shih YF, Hsiao CK, Chen CJ. Prevalence of myopia in Taiwanese schoolchildren: 1983 to 2000. Ann Acad Med Singapore. 2004;33:27-33.

2. Lyle WA, Jin GJ. Retreatment after initial laser in situ keratomileusis. J Cataract Refract Surg. 2000;26:650-659.

3. Chayet AS, Assil KK, Montes M, et al. Regression and its mechanisms after laser in situ keratomileusis in moderate and high myopia. Opbthalmology. 1998.;105:1194-1199.

4. Hu DJ, Feder RS, Basti S, et al. Predictive formula for calculating the probability of LASIK enhancement. J Cataract Refract Surg. 2004; 30:363-368.

5. Albietz JM, Lenton LM, McLennan SG. Chronic dry eye and regression after laser in situ keratomileusis for myopia. J Cataract Refract Surg. 2004;30:675-684

6. Lian J, Zhang Q, Ye W, Zhou D, Wang K. An analysis of regression after laser in situ keratomileusis for treatment of myopia. Zhongbua Yan Ke Za Zbi. 2002;38:363-366.

7. Condon PI, Mulhern M, Fulcher T, Foley-Nolan A, O'Keefe M. Laser intrastromal keratomileusis for high myopia and myopic astigmatism. Br J Opbthalmol. 1997;81:199-206.

8. Patel NP, Clinch TE, Weis JR, Ahn C, Lundergan MK, Heidenreich $\mathrm{K}$. Comparison of visual results in initial and re-treatment laser in situ keratomileusis procedures for myopia and astigmatism. Am J Opbthalmol. 2000;130:1-11.

9. Ozdamar A, Aras C, Bahcecioglu H, Sener B. Secondary laser in situ keratomileusis 1 year after primary LASIK for high myopia. $J$ Cataract Refract Surg. 1999;25:383-388.

10. Gimbel HV, Penno EE, van Westenbrugge JA, Ferensowicz M, Furlong MT. Incidence and management of intraoperative and early postoperative complications in 1000 consecutive laser in situ keratomileusis cases. Opbthalmology. 1998;105:1839-1847.
11. Collett, D. Modelling Survival Data in Medical Research. 2nd ed. Bristol, UK: Taylor \& Francis; 2003:237-251.

12. Ditzen K, Handzel A, Pieger S. Laser in situ keratomileusis nomogram development. J Refract Surg. 1999;15:S197-S201.

13. Feltham MH, Wolfe RJ. Some variables to consider to avoid the need for LASIK surgical enhancements. Clin Exp Optom. 2000;83: $76-81$.

14. Rao SK, Cheng AC, Fan DS, Leung AT, Lam DS. Effect of preoperative keratometry on refractive outcomes after laser in situ keratomileusis. J Cataract Refract Surg. 2001;27:297-302.

15. Pan Q, Gu YS, Wang J, et al. Differences between regressive eyes and non-regressive eyes after LASIK for myopia in the time course of corneal changes assessed with the Orbscan. Opbthalmologica. 2004;218:96-101.

16. Perez-Santonja JJ, Bellot J, Claramonte P, Ismail MM, Alio JL. Laser in situ keratomileusis to correct high myopia. J Cataract Refract Surg. 1997;23:372-385.

17. Pallikaris IG, Siganos DS. Excimer laser in situ keratomileusis and photorefractive keratectomy for correction of high myopia. $J \mathbf{R e}$ fract Corneal Surg. 1994;10:498-510.

18. Zaldivar R, Davidorf JM, Shultz MC, Oscherow S. Laser in situ keratomileusis for low myopia and astigmatism with a scanning spot excimer laser. J Refract Surg. 1997;13:614-619.

19. Pallikaris IG, Siganos DS. Laser in situ keratomileusis to treat myopia: early experience. J Cataract Refract Surg. 1997;23:3949.

20. Magallanes R, Shah S, Zadok D, et al. Stability after laser in situ keratomileusis in moderately and extremely myopic eyes. J Cataract Refract Surg. 2001;27:1007-1012.

21. Zheng L, Zhu Q, Sun Y, Ye M. The complications of excimer laser in-situ keratomileusis for myopia. Zbonghua Yan Ke Za Zhi. 1999;35:363-365.

22. Farah SG, Azar DT, Gurdal C, Wong J. Laser in situ keratomileusis: literature review of a developing technique. J Cataract Refract Surg. 1998;24:989-1006.

23. No authors listed. Induced astigmatism following laser in situ keratomileusis for myopia with a free cap. J Refract Surg. 2000; 16:375-379.

24. Eleftheriadis H, Prandi B, Diaz-Rato A, Morcillo M, Sabater JB. The effect of flap thickness on the visual and refractive outcome of myopic laser in situ keratomileusis. Eye. 2005;19:1290-1296.

25. Wilson SE, Mohan RR, Hong JW, Lee JS, Choi R, Mohan RR. The wound healing response after laser in situ keratomileusis and photorefractive keratectomy: elusive control of biological variability and effect on custom laser vision correction. Arch Opbthalmol. 2001;119:889-896.

26. Perez-Santonja JJ, Ayala MJ, Sakla HF, Ruiz-Moreno JM, Alio JL. Retreatment after laser in situ keratomileusis. Ophthalmology. 1999;106:21-28. 